\title{
A practical method for predicting frequent use of emergency department care using routinely available electronic registration data
}

\author{
Jianmin $\mathrm{Wu}^{1 *}$, Shaun J. Grannis ${ }^{1 *}$, Huiping $X \mathrm{u}^{2}$ and John T. Finnell ${ }^{1}$
}

\begin{abstract}
Background: Accurately predicting future frequent emergency department (ED) utilization can support a case management approach and ultimately reduce health care costs. This study assesses the feasibility of using routinely collected registration data to predict future frequent ED visits.

Method: Using routinely collected registration data in the state of Indiana, U.S.A., from 2008, we developed multivariable logistic regression models to predict frequent ED visits in the subsequent two years. We assessed the model's accuracy using Receiver Operating Characteristic (ROC) curves, sensitivity, and positive predictive value (PPV).

Results: Strong predictors of frequent ED visits included age between 25 and 44 years, female gender, close proximity to the ED (less than 5 miles traveling distance), total visits in the baseline year, and respiratory and dental chief complaint syndromes. The area under ROC curve (AUC) ranged from 0.83 to 0.92 for models predicting patients with 8 or more visits to 16 or more visits in the subsequent two years, suggesting acceptable discrimination. With $25 \%$ sensitivity, the model predicting frequent ED use as defined as 16 or more visits in 2009 and 2010 had a PPV of $59.5 \%$ and specificity of $99.9 \%$. The "adjusted" PPV of this model, which includes patients having 8 or more visits, is $81.9 \%$.

Conclusion: We demonstrate a strong association between predictor variables present in registration data and frequent ED use. The algorithm's performance characteristics suggest that it is technically feasible to use routinely collected registration data to predict future frequent ED use.
\end{abstract}

Keywords: Emergency department, Registration data, Prediction, Frequent use

\section{Background}

With increasing medical costs, health care reformers and policy makers have turned to emergency department (ED) utilization as a potential source for cost savings. A relatively small number of patients, often called "frequent" or "high ED users", have been an increasing focus because of their disproportionate share of ED visits and cost. When defined as 4 or more ED visits per year, frequent users accounted for 4.5 to $8 \%$ of all ED patients and contributed 21 to $28 \%$ of all ED visits [1]. Prior interventions targeting frequent users did not achieve universally positive outcomes although some studies

\footnotetext{
* Correspondence: jianminwu2014@gmail.com; sgrannis@regenstrief.org ${ }^{1}$ Regenstrief Institute, Inc., 1101 West 10th Street, Indianapolis, IN 46202, USA Full list of author information is available at the end of the article
}

demonstrate reduced ED use [2-6]. A clear framework including a consensus-based definition of frequent users and methods to accurately and consistently identify this population may improve the effectiveness of care management interventions.

Currently, however, a standardized definition for frequent ED users remains elusive. A single visit threshold has been used to differentiate frequent users from low ED users, and the visit threshold varies from as few as 3 to 12 or more annual visits, often without a clear rationale for the visit cut-point [7-13]. Further, the majority of prior studies on frequent ED users focused on identifying existing frequent ED users [7, 9, 11, 14], which is problematic as most frequent users in a given year will not remain frequent users in the next year. It has been 
shown that an individual who has 4 or more visits in a given year was only 28 to $38 \%$ likely to be a frequent user the next year [1]. Fertel et al. also showed that highly frequent use occurs for only a minority of ED patients, and then only for a discrete period [15]. Roland et al. pointed out that the "regression to mean" phenomenon should not be ignored when evaluating interventions for frequent ED users [16]. Therefore, blindly targeting most current frequent ED users for future interventions is inefficient because their heavy use of ED services may decrease without intervention. Since health care resources are limited, it is essential that interventions target patients whose heavy ED use will likely persist. Thus, the capacity to predict patients who are likely to sustain frequent future ED utilization can help address this problem by identifying patients who are most likely to generate future heavy ED use and costs.

Previously we reported that 2.8 million patients from 96 EDs in the state of Indiana within United States generated 7.4 million ED visits from 2008 to 2010, and the average number of visits was 2.6 visits per patient [17]. We found that patients cross over to other ED institutions with great frequency, and about $3.3 \%$ of the patients made more than 10 visits to Indiana EDs from 2008 to 2010 [17]. In this study, we explore whether specific features contained within routinely gathered registration data could meaningfully predict a patient's future ED utilization. If these features accurately predict future frequent ED users, then we can more effectively, target limited health care resources on this group, maximizing the benefit of the intervention. The purpose of this study is to assess the feasibility of using routinely gathered registration data to predict patients who will visit ED's with high frequency.

\section{Methods}

This study was approved by Indiana State Department of Health (ISDH) data release committee and the Indiana University Institutional review board (USA).

\section{Datasets}

Data collected for this study were derived from the original Health Level-7 (HL7) version 2 registration transactions for ED encounters from 96 institutions participating in the Indiana Public Health Emergency Surveillance System (PHESS) between January 1, 2008 and December 31,2010 . The data is not publically available but can be accessed through the Regenstrief Institute Data Core (https://www.regenstrief.org/hsr/research-programs/rcher/ data-core/).

The processes for preparing ED encounter data as well as the details for each step were presented in our previous paper [17]. Briefly, registration transactions were processed to ensure each transaction was unique and contained valid
ED encounter data according to PHESS requirements and a set of heuristics drawn from Regenstrief's long-term realworld experience operating a health information exchange. Unique ED encounters were established using data elements including person, place and time. The specific fields included [1] healthcare institution (HL7 MSH-4), [2] ED encounter date (HL7 PV1-44), and [3] medical record number (HL7 PID-3). Transactions missing any of these fields could not be definitively and uniquely identified as an encounter and were excluded from the analysis.

Unique patients were identified using various combinations of patient demographics, including social security number, last and first name, gender, date of birth, telephone number, and zip code as determined by an open-source probabilistic record linkage software package [18]. In this manner all ED encounters belonging to the same patient were linked, forming a "patient group." A unique global patient identifier was assigned to each patient group. In total, we identified 7,447,521 unique ED encounters. Data available for analysis includes: age, sex, chief complaints, ZIP codes for patients' address, and hospital ZIP codes. Patients' global identifier was used to link visits across different hospital databases, including all ED visits regardless of disposition.

\section{Predictive model}

We developed multivariable logistic regression models. Patients with at least one ED visit in 2008 were used to predict ED visits in the years of 2009 and 2010. Patients who died before January 1, 2009 or had missing values in one or more covariates were excluded $(<4.30 \%)$. The final sample size was $1,272,367$ patients. All variables were summarized at the patient level for model development.

\section{Covariates}

All covariates were determined based on the ED utilization data in 2008.

Age: age was determined at the time of the first ED visit, and divided into six subgroups: $<5,5-14,15-24$, $25-44,45-64$ and $>=65$ years.

Sex: male and female;

Visits in 2008: the total number of ED visits made in 2008 for each patient;

Chief complaints: the chief complaint syndromes were grouped into 11 categories: respiratory, gastrointestinal (GI), undifferentiated infection (UDI), influenza-like illness (ILI), lymphatic, skin, neurological, pain, dental, alcohol and musculoskeletal syndromes. These categories were used by other surveillance programs with slight modification [19-21]. Chief complaints that could not be grouped into the above 11 syndromes were assigned to "unclassified". The categories were then reviewed by two physicians (Grannis S, Finnel JT) and an epidemiologist. For each patient, the proportion of each chief complaint 
syndrome is determined through dividing the number of ED visits with a specific syndrome by the total number of ED visits that the patient had in 2008. Since one ED visit may have more than one syndrome, these percentages do not add up to $100 \%$.

Zip code centroid straight-line distances: The Perl library Geo::Distance was used to calculate the straightline distances between geographic points from patients' home to hospital based on zip code centroids of patient's home address and hospital address. Distance was then grouped into 3 categories: <=5 miles, 5-20 miles and $>20$ miles. Since one patient may have multiple ED visits with different distance, we determined the proportion of ED visits falling into each of the three categories by dividing the number of ED visits with a specific distance category by the total number of ED visits that a patient made in 2008. Because the proportions for each of these three distance categories add up to $100 \%$, only two categories $(<5$ miles and $>20$ miles) were included in the analytic model.

\section{Study outcome}

The outcome was measured as dichotomized variable (frequent versus low ED user). Frequent ED users were investigated by using visit cut-points ranging from 8 to 16 visits over a two-year period (between 2009 and 2010). One model was fit for each cut point. Patients were defined as frequent ED users if their ED visits were equal to or higher than the visit cut-point, and were otherwise defined as low ED users.

\section{Model performance evaluation}

The model's performance was assessed for discrimination using the Receiver Operating Characteristic (ROC) curves. We balanced the goal of identifying all frequent ED utilizers with the intervention cost of incorrectly identifying frequent ED users by selecting a fixed sensitivity of $25 \%$ to minimize the false positive rate. We then evaluated the specificity and positive predictive value (PPV) for each model at fixed sensitivity of $25 \%$. We also combined the false positive (FP) patients who had 8 or more visits with the true positive (TP) patients to obtain the "adjusted" positive cohort. The "adjusted" PPV was determined by dividing the "adjusted" positive group by the sum of TP and FP. Statistical analyses were conducted using SAS version 9.3 (SAS Corporation; Cary, North Carolina).

\section{Results}

\section{ED utilization and patients' distribution by year}

During the 3-year study period, 2.8 million patients generated 7.4 million ED visits. Of patients with ED visits in 2008, about one-fifth came back to the ED in both 2009 and 2010 and one-third of them returned either in 2009 or in 2010. Almost half did not present to ED in the following two years (Fig. 1a). Interestingly, patients who made 4 or more visits in 2008 showed a similar pattern. $8.5 \%$ of all 2008 patients had 4 or more visits in 2008 . But only $35 \%$ and $27 \%$ of them continued to have 4 or more visits in 2009 and 2010 respectively. The majority returned to low or no ED use in the subsequent two years (Fig. 1b). Interestingly, about $4 \%$ of patients with less than 4 visits in 2008 increased their ED utilization to 4 or more visits either in 2009 or 2010 (Fig. 1c).

\section{Characteristics of ED patients}

Patients aged 25 to 44 years were the largest group and accounted for $\sim 29 \%$ of the total visits and $\sim 26 \%$ of total patients. The second largest age group were 44 to 65 years and contributed $\sim 20 \%$ of the total visits. Patients aged 5 to 14 years had the least number of visits and accounted for 8 to $9 \%$ of total visits in each year, respectively. More than $53 \%$ of patients were female and they accounted for $55 \%$ of visits.

The distribution of patients with ED visits equal to or greater than 4, 8, and 16 annually is presented in Table 1 . Patients with 4 or more visits per year accounted for 30 to $45 \%$ of the total visits each year. Although the patients who had 16 visits annually only accounted for $0.3 \%$ of the total patients, they contributed almost $4 \%$ of the total visits.

The profile of ED visits by the zip code centroid straight-line distance between home and hospital in each year is very similar. More than $60 \%$ of ED visits were associated with a distance of 5 miles or less. About $30 \%$ of visits had a distance between 5 and 20 miles, and $8 \%$ of visits had distance of more than 20 miles (Table 2).

Table 2 captures chief complaint distributions. "Pain" was the most common chief complaint category, accounting for more than $40 \%$ of total visits, while the "musculoskeletal" category contributed $\sim 28 \%$ of total ED visits. "Respiratory" and "gastrointestinal" syndrome categories accounted for more than $23 \%$ and $17 \%$ of total visits, respectively. The "alcohol" accounted for $0.5 \%$ of total visits each year. Nearly $15 \%$ of visits were grouped into the "unclassified" category.

\section{Multivariable logistic regression model predicting frequent use of $E D$ care}

We developed logistic regression models to predict the likelihood for a patient to be a frequent ED user in years 2009 and 2010 based on the 2008 registration data. Table 3 presents the patients' characteristics used in the models stratified by number of visits in 2009 and 2010 . Overall, common characteristics of frequent ED users include age between 15 and 44 years, female gender, and utilizing ED care frequently in the baseline year. They 

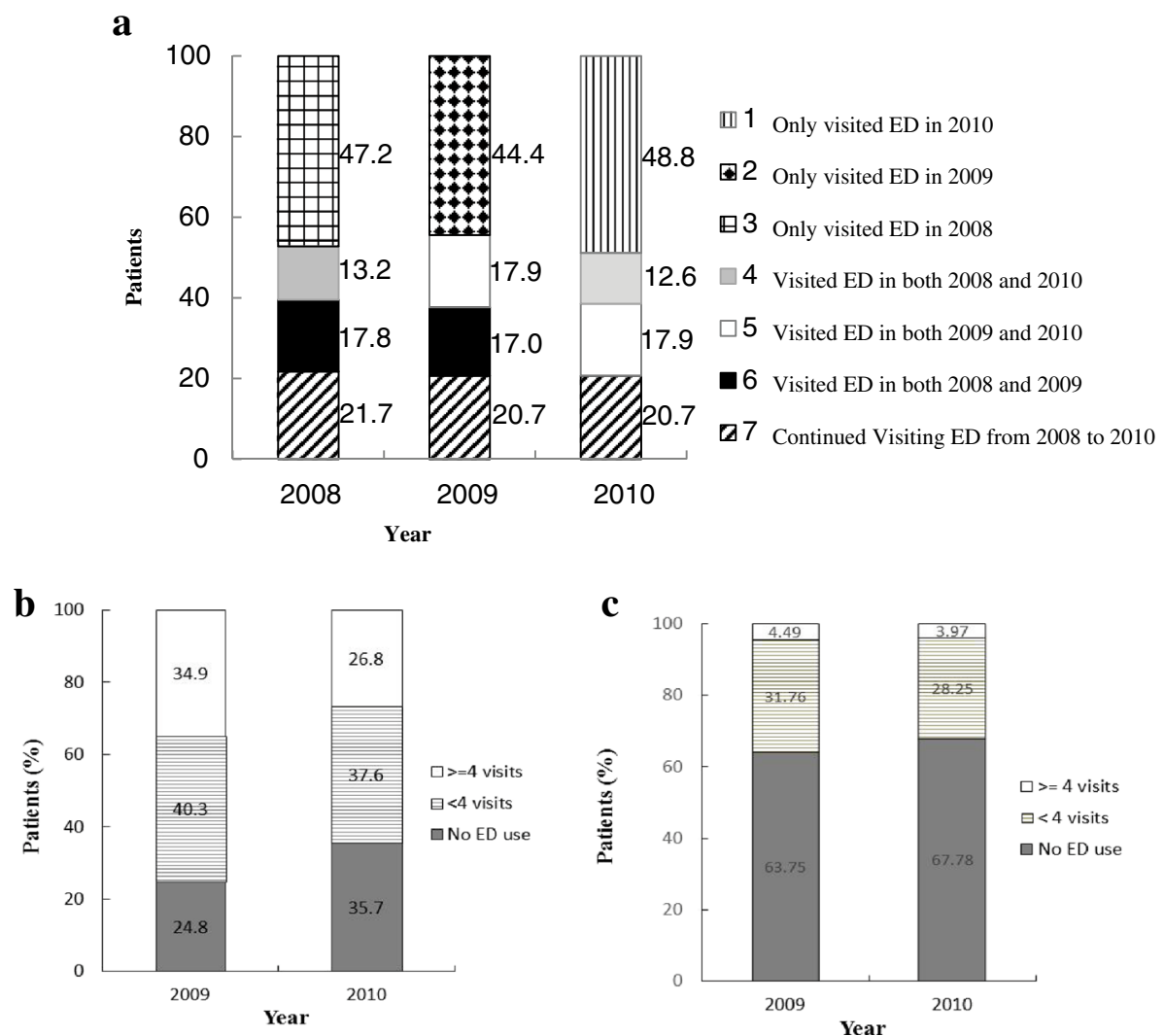

Fig. 1 a. Distribution of patients stratified by year, 2008 to 2010; b. Emergency department utilization in 2009 and 2010 for patients with 4 or more visits in 2008; c. Emergency department utilization in 2009 and 2010 for patients with fewer visits less than 4 in 2008

commonly report "pain", "musculoskeletal", "respiratory", and/or "gastrointestinal" syndromes. More than $77 \%$ of frequent ED users had one or more visits with a travel distance equal to or less than 5 miles. Table 4 shows the results from two logistic regression models predicting frequent use of ED with 8 or more visits, and frequent use of ED with 16 or more visits, respectively. Age, sex, zip code centroid straight-line distance between home and hospital, and specific chief complaints, including respiratory, dental, and alcohol syndromes were predictive for frequent use of the emergency room in 2009 and 2010. The

Table 1 Distribution of patients and ED visits by visit cut-points in each year

\begin{tabular}{llccc}
\hline & Year (Pat. No.) & \multicolumn{3}{l}{ Visits cut-point } \\
\cline { 3 - 5 } & & $>=4$ & $>=8$ & $>=16$ \\
\hline Patients (\%) & $2008(n=1329645)$ & 8.5 & 1.6 & 0.3 \\
& $2009(n=1396313)$ & 9.2 & 1.7 & 0.3 \\
& $2010(n=1397338)$ & 8.9 & 1.6 & 0.3 \\
Visits (\%) & $2008(n=2367399)$ & 29.8 & 11.0 & 3.7 \\
& $2009(n=2551881)$ & 31.3 & 11.6 & 3.9 \\
& $2010(n=2528241)$ & 45.1 & 21.0 & 3.9 \\
\hline
\end{tabular}

variable representing number of visits in 2008 plus a quadratic term to account for some non-linearity in age associations led to a better fit of the model, and was highly predictive for frequent use of ED the following two years. The significance of the predictors is consistent across these models, and the coefficients for most of the predictors revealed a similar profile. The equations for these two logistic regression models are summarized in Fig. 2.

We next evaluated the model's performance using ROC curves, specificity, and PPV (Table 5). The model predicting frequent ED use with 8 or more visits in the subsequent two years showed satisfactory discriminating power with an AUC of 0.84 (Model No.1). The model predicting frequent ED use as defined as 16 or more visits in the subsequent two years showed better discrimination, with AUC of 0.92 (Model No. 9). We also explored patient characteristics among the false positive (FP) and false negative (FN) groups. Interestingly, patients in the FP group had an equal number of visits in the 2008 baseline year when compared to the true positive (TP) patients (Mean visits of FP vs TP: $11.0 \pm 4.3$ vs $14.1 \pm 7.9$ for Model No. $1 ; 18.9 \pm 6.8$ vs $22.8 \pm 9.8$ for Model No. 9). However, when compared with the TP cohort, the FP patients yielded a lower proportion aged 
Table 2 Distribution of ED visits by travel distance and chief complaints in each year, 2008 to 2010

\begin{tabular}{lccc}
\hline Characteristics & $2008(\%)$ & $2009(\%)$ & $2010(\%)$ \\
\hline Travel distance (miles) & & & \\
$<=5$ & 60.1 & 60 & 60.1 \\
$5-20$ & 30.2 & 30.7 & 30.3 \\
$>20$ & 8.4 & 8.1 & 8.3 \\
Missing & 1.4 & 1.2 & 1.3 \\
Chief complaint & & & \\
Respiratory & & 24.6 & 22.0 \\
Gastrointestinal & 17.1 & 17.6 & 17.7 \\
Neurologic & 11.3 & 11.2 & 11.2 \\
Skin & 4.6 & 4.4 & 4.7 \\
UDI & 9 & 10.5 & 8.8 \\
Lymphatic & 3 & 3.0 & 3.0 \\
ILI & 18.8 & 20.7 & 20.9 \\
Dental & 1.8 & 1.8 & 1.8 \\
Pain & 41.5 & 40.3 & 40.8 \\
Musculoskeletal & 28.7 & 27.5 & 27.7 \\
Alcohol & 0.5 & 0.5 & 0.6 \\
Unclassified & 15.7 & 14.8 & 15.4 \\
Missing & 1.34 & 1.47 & 3.41 \\
\hline Because & & & \\
\hline
\end{tabular}

${ }^{\mathrm{a}}$ Because one visit may have multiple syndromes, the total percentage of chief complaints is more than $100 \%$

$15-24$ year, and a higher proportion $>=65$ years, fewer were female, and there was a lower proportion of "respiratory" chief complains. Regarding the FN group, their total number of visit in the baseline year was comparable to the true negative (TN) group (Mean visits of FN vs TN: $3.3 \pm 3.2$ vs $1.5 \pm 1.0$ for Model No. $1 ; 6.08 \pm$ 6.3 vs $1.7 \pm 1.3$ for Model No. 9). The FN group also exhibited a larger proportion of "respiratory" and "alcohol" complaints; more were aged 25-44 years, and more were associated with a travel distance within 5 miles when compared to the TN group. Finally, we evaluated the specificity and PPV of the models at $25 \%$ sensitivity. Model No. 9 which predicts frequent ED use as defined as 16 or more visits in 2009 and 2010, revealed a PPV of $59.5 \%$ and specificity of $99.9 \%$. A significant proportion of FP patients visited the ED repeatedly although they did not meet the specific threshold of frequent ED use. For example, $55.3 \%$ of FP patients had 8 or more visits in Model No. 9, and the "adjusted" PPV, which included patients having 8 or more visits in the subsequent two years as positives targets, was $81.9 \%$.

\section{Discussion}

The primary goals of this study were to evaluate the feasibility of using routinely available registration data to predict patients likely to use ED services frequently in
Table 3 Distribution of predictors in 2008 stratified by number of visits in 2009 and 2010

\begin{tabular}{|c|c|c|c|c|c|}
\hline & \multicolumn{4}{|c|}{ Visits in 2009 \& 2010} & \multirow{2}{*}{$\begin{array}{l}\text { Total } \\
\text { (Patients No.) }\end{array}$} \\
\hline & 0 & $1-7$ & $8-15$ & $>=16$ & \\
\hline $\begin{array}{l}\text { Year } 2008 \\
\text { patients (No.) }\end{array}$ & 587523 & 623355 & 47782 & 13707 & 1272367 \\
\hline \multicolumn{6}{|c|}{ Age (Years) (Patients, \%) } \\
\hline $15-24$ & 44.1 & 49.2 & 5.2 & 1.5 & 206230 \\
\hline $25-44$ & 45.9 & 47.4 & 4.8 & 1.9 & 346349 \\
\hline $45-64$ & 50.5 & 44.9 & 3.5 & 1.1 & 262691 \\
\hline $5-14$ & 51.6 & 46.8 & 1.4 & 0.1 & 137222 \\
\hline$<5$ & 43.7 & 53.6 & 2.5 & 0.2 & 131743 \\
\hline$>=65$ & 40.6 & 55.7 & 3.3 & 0.4 & 188132 \\
\hline \multicolumn{6}{|c|}{ Sex (Patients, \%) } \\
\hline Male & 49.6 & 46.6 & 2.9 & 0.8 & 590065 \\
\hline Female & 43.2 & 51.1 & 4.5 & 1.3 & 682302 \\
\hline \multicolumn{6}{|c|}{ Visits in 2008 (Patients, \%) } \\
\hline$<4$ & 49.2 & 48.2 & 2.3 & 0.3 & 1167719 \\
\hline $4-7$ & 14.6 & 62.8 & 17.8 & 4.8 & 85359 \\
\hline $8-15$ & 6.2 & 37.9 & 31.2 & 24.7 & 15964 \\
\hline$>=16$ & 2.7 & 13.1 & 19.9 & 64.3 & 3325 \\
\hline \multicolumn{6}{|c|}{ Chief complaints in 2008 (Visits, \%) } \\
\hline RESP & 62.9 & 28.7 & 5.1 & 3.3 & 976602 \\
\hline $\mathrm{Gl}$ & 32.2 & 50.6 & 9.6 & 7.6 & 390479 \\
\hline NEURO & 34.4 & 51.6 & 8.4 & 5.6 & 248617 \\
\hline SKIN & 35.8 & 54.0 & 7.2 & 3.1 & 105329 \\
\hline LYMP & 33.3 & 52.8 & 8.9 & 5.1 & 65627 \\
\hline UDI & 33.5 & 56.3 & 7.0 & 3.2 & 207325 \\
\hline PAIN & 34.7 & 49.4 & 9.1 & 6.9 & 953772 \\
\hline ILI & 33.0 & 54.3 & 8.1 & 4.6 & 429244 \\
\hline DENTAL & 24.7 & 51.5 & 13.8 & 10.0 & 41990 \\
\hline MUSC & 37.8 & 49.2 & 7.9 & 5.1 & 654713 \\
\hline $\mathrm{ETOH}$ & 33.7 & 43.8 & 11.1 & 11.4 & 12286 \\
\hline OTHER & 37.3 & 50.0 & 7.8 & 4.8 & 353451 \\
\hline \multicolumn{6}{|c|}{ Distance (Miles) (Visits, \%) } \\
\hline$<=5$ & 34.8 & 63.1 & 1.6 & 0.4 & 952546 \\
\hline $5-20$ & 42.7 & 55.9 & 0.8 & 0.6 & 618869 \\
\hline$>20$ & 56.5 & 41.9 & 0.4 & 1.2 & 173725 \\
\hline
\end{tabular}

the future and to develop strategies for improving the accuracy and efficiency of detecting frequent ED users. We demonstrate a strong association between predictor variables present in routine registration data and frequent ED use. The algorithm's performance characteristics suggest that it is technically feasible to use routinely collected registration data to predict such use, and the model's observed prediction accuracy may support identifying and intervening upon frequent ED users. Thus, 
Table 4 Multivariable logistic regression models predicting frequent ED users having $>=8$ visits and frequent ED users having $>=16$ visits in 2009 and 2010

\begin{tabular}{|c|c|c|c|c|c|c|}
\hline \multirow{2}{*}{$\begin{array}{l}\text { Predicting } \\
\text { variable }\end{array}$} & \multicolumn{3}{|l|}{$>=8$ visits } & \multicolumn{3}{|l|}{$>=16$ visits } \\
\hline & Coefficient & Standard error & $p$ value & Coefficient & Standard error & $p$ value \\
\hline \multicolumn{7}{|l|}{ Age (year) } \\
\hline $5-14$ & Ref & & & & & \\
\hline$<5$ & 0.2823 & 0.0292 & $<.0001$ & 0.0763 & 0.1024 & 0.4562 \\
\hline $15-24$ & 1.0897 & 0.0248 & $<.0001$ & 1.7732 & 0.0801 & $<.0001$ \\
\hline $25-44$ & 0.9673 & 0.0241 & $<.0001$ & 1.7804 & 0.079 & $<.0001$ \\
\hline $45-64$ & 0.7146 & 0.0252 & $<.0001$ & 1.4661 & 0.0805 & $<.0001$ \\
\hline$>=65$ & 0.5033 & 0.0266 & $<.0001$ & 0.6751 & 0.0864 & $<.0001$ \\
\hline Visits in 2008 & 0.5829 & 0.00243 & $<.0001$ & 0.5048 & 0.00319 & $<.0001$ \\
\hline$\left(\right.$ Visits in 2008) ${ }^{2}$ & -0.0075 & 0.00011 & $<.0001$ & -0.0062 & 0.00011 & $<.0001$ \\
\hline \multicolumn{7}{|l|}{ Sex (ref. = "Male") } \\
\hline Male & Ref. & & & & & \\
\hline Female & 0.3173 & 0.00978 & $<.0001$ & 0.2663 & 0.0212 & $<.0001$ \\
\hline \multicolumn{7}{|c|}{ Travel Distance (miles) } \\
\hline $5-20$ & Ref. & & & & & \\
\hline$<=5$ & 0.344 & 0.0115 & $<.0001$ & 0.3263 & 0.0259 & $<.0001$ \\
\hline$>20$ & -0.2396 & 0.023 & $<.0001$ & -0.0685 & 0.0501 & 0.171 \\
\hline \multicolumn{7}{|l|}{ Chief Complaints } \\
\hline Respiratory & 0.4202 & 0.0203 & $<.0001$ & 0.4527 & 0.0487 & $<.0001$ \\
\hline Gastrointestinal & 0.0783 & 0.019 & $<.0001$ & 0.1195 & 0.0437 & 0.0062 \\
\hline Neurologic & 0.1084 & 0.021 & $<.0001$ & 0.1487 & 0.049 & 0.0024 \\
\hline Skin & -0.0983 & 0.0317 & 0.002 & -0.4246 & 0.0858 & $<.0001$ \\
\hline UDI & 0.00071 & 0.0254 & 0.9777 & -0.0999 & 0.0648 & 0.123 \\
\hline Lymphatic & 0.066 & 0.0361 & 0.0679 & -0.0848 & 0.0896 & 0.344 \\
\hline ILI & -0.2013 & 0.0201 & $<.0001$ & -0.3398 & 0.0489 & $<.0001$ \\
\hline Dental & 0.4419 & 0.0393 & $<.0001$ & 0.372 & 0.0843 & $<.0001$ \\
\hline Pain & 0.081 & 0.0153 & $<.0001$ & 0.2139 & 0.0359 & $<.0001$ \\
\hline Musculoskeletal & -0.1625 & 0.0186 & $<.0001$ & -0.2645 & 0.0434 & $<.0001$ \\
\hline Alcohol & 0.2296 & 0.0753 & 0.0023 & 0.4842 & 0.1447 & 0.0008 \\
\hline Unclassified & -0.1018 & 0.0241 & $<.0001$ & -0.0771 & 0.0574 & 0.1789 \\
\hline
\end{tabular}

\section{Model A}

$\log ($ Odds $)=-5.57+0.28 * \operatorname{Age}(<5)+1.09 * \operatorname{Age}(15-24)+0.97 * \operatorname{Age}(25-44)+0.71 * \operatorname{Age}(45-64)$

$+0.50 *$ Age $(>=65)+0.32 *$ Female $+0.34 *$ Distance $(<=5)-0.24 *$ Distance $(>20)$

$+0.58 *$ Visits_2008 $-0.0075 *(\text { Visits_2008 })^{2}+0.08 *$ CC_GI $-0.10 *$ CC_Skin

$+0.42 *$ CC_RESP $+0.11 *$ CC_NEURO $+0.001 *$ CC_UDI $-0.20 *$ CC_ILI $+0.08 *$ CC_Pain

$+0.44 *$ CC_Dental $-0.16 *$ CC_MUSC $+0.07 *$ CC_Lymphatic $+0.23 *$ CC_Alcohol $-0.10 *$ CC_Unclassified

Model B

$\log ($ Odds $)=-7.93+0.08 * \operatorname{Age}(<5)+1.77 * \operatorname{Age}(15-24)+1.78 * \operatorname{Age}(25-44)+1.47 * \operatorname{Age}(45-64)$

$+0.68 *$ Age $(>=65)+0.27 *$ Female $+0.33 *$ Distance $(<=5)-0.069 *$ Distance $(>20)$

$+0.50 *$ Visits $2008-0.0062 *$ (Visits 2008$)^{2}+0.12 *$ CC GI $-0.42 *$ CC S Skin $+0.45 *$ CC RESP

$+0.15 *$ CC_NEURO $-0.10 *$ CC_UDI $-0.34 *$ CC_ILI $+0.21 *$ CC_Pain $+0.37 *$ CC_Dental

$-0.26 *$ CC_MUSC $-0.08 *$ CC_Lymphatic $+0.48 *$ CC_Alcohol $-0.08 *$ CC_Unclassified

Fig. 2 Equations for model predicting frequent emergency department (ED) use as defined as 8 or more visits (a) and model predicting frequent ED use as defined as 16 or more visits in the subsequent two years $(\mathbf{b})$. Distance $(<=5)$ : the straight-line distances between geographic points from patients' home to hospital less than 5 miles; Distance (>20): the straight-line distances between geographic points from patients' home to hospital greater than 20 miles; CC: chief complaints; GI: gastrointestinal; RESP: respiratory; NEURO: neurological; UDI: undifferentiated infection; ILI: influenza-like illness and MUSC: musculoskeletal 
Table 5 Model evaluation

\begin{tabular}{|c|c|c|c|c|c|c|c|c|c|c|}
\hline \multirow{2}{*}{ Model No. } & & \multicolumn{9}{|c|}{ Multivariable Logistic Regression Models } \\
\hline & & 1 & 2 & 3 & 4 & 5 & 6 & 7 & 8 & 9 \\
\hline \multicolumn{2}{|c|}{ No. of visits constituting 'frequent use' } & $>=8$ & $>=9$ & $>=10$ & $>=11$ & $>=12$ & $>=13$ & $>=14$ & $>=15$ & $>=16$ \\
\hline \multicolumn{2}{|c|}{ Area under ROC curve (AUC) } & 0.84 & 0.85 & 0.87 & 0.88 & 0.89 & 0.89 & 0.90 & 0.91 & 0.92 \\
\hline \multicolumn{11}{|c|}{ With sensitivity $<=25 \%$, probability $>0.5$} \\
\hline \multicolumn{2}{|l|}{ PPV (\%) } & 64.5 & 63.9 & 63.4 & 62.9 & 62.4 & 61.3 & 60.8 & 60.6 & 59.5 \\
\hline \multicolumn{2}{|l|}{ Specificity (\%) } & 99.5 & 99.6 & 99.7 & 99.7 & 99.8 & 99.8 & 99.8 & 99.8 & 99.9 \\
\hline \multirow[t]{2}{*}{ False positive patients } & Total No. & 5883 & 4923 & 4125 & 3447 & 2974 & 2610 & 2273 & 2007 & 1805 \\
\hline & $>=8$ visits (No.) & 0 & 565 & 843 & 1021 & 1071 & 1103 & 1077 & 1038 & 998 \\
\hline \multicolumn{2}{|c|}{$\begin{array}{l}\text { Adjusted PPV for patients with > }=8 \\
\text { visits in subsequent two years (\%) }\end{array}$} & 64.5 & 68 & 70.9 & 73.9 & 75.9 & 77.7 & 79.4 & 81 & 81.9 \\
\hline
\end{tabular}

such models may support more effective targeting of limited health care resources to patients who may maximally benefit from intervention.

Much of the literature studying frequent ED utilization has substantial limitations, which our study sought to address. First, some published studies used data from a limited number of ED's and thus their broad generalizability is unclear [7-12, 22, 23]. Although several statewide studies in United States explored ED visits across age, gender, health insurance groups and clinical characteristics between frequent and in-frequent ED users, most were descriptive in nature and few applied prediction models to identify frequent ED users [24-27]. Second, some studies used survey or interview data and the quality and reliability of such data can be affected by survey response rates [8-10]. Further, the cost, time and other resources involved in the interview may be prohibitive. Third, some studies focused on specific cohorts such as asthmatics or the elderly, and this limits the ability of policy makers and providers to determine whether unifying factors that could be targeted for intervention exist amongst a more general population of patients with frequent ED utilization. Lastly and most importantly, in many cases researchers focused on identifying existing frequent ED users instead of predicting future frequent ED utilization [7, 9, 11, 14]. As shown in ours and others studies $[1,15,16]$, most patients do not remain frequent ED users over time and many naturally reduce their ED use without intervention (regression toward mean). Thus, predicting patients who are likely to sustain future frequent ED utilization will be necessary for improving the health of this vulnerable patient group.

Developing algorithms that accurately identify patients who are likely to frequently visit ED's in subsequent years is a first step toward developing potential interventions to mitigate overuse. However, few studies have leveraged any approach or method to identify future frequent ED users [4,28-31]. In those studies, frequent ED users were defined with a threshold number of ED visits, e.g. 3 to $10 \mathrm{ED}$ visits within the 12 months prior to the study period. In addition, the majority of the comparative cohort studies used a pre-and post-intervention design, where the population exposed to the intervention served as their own historical control groups, without recognizing the regression toward mean phenomenon, which might incorrectly inflate the effectiveness of interventions.

In our study, we developed a practical approach to predict future frequent ED users. The model predicting patients with 8 or more visits in the subsequent two years demonstrated reasonable discriminative power with an AUC of 0.84. As the threshold defining 'frequent use' increases, the corresponding AUC also increased. The model predicting frequent ED use of 16 or more visits in the subsequent two years showed good discrimination, with an AUC of 0.92. Strong predictor variables included visits in the baseline year, age, sex, zipcode centroid straight-line distance between home and hospital, and specific chief complaints, including respiratory, dental and alcohol syndromes. When comparing false positives to true positives and false negatives to true negatives, respectively, we noted that the variable "Number of visits in the baseline year" were very close, indicating that patients' other features contained within routinely gathered registration data contributed additional discriminating power.

If the algorithm incorrectly flags patients as frequent utilizers, the resulting inefficiencies may offset potential savings from subsequent reduced ED utilization. Considering the trade-offs between (a) identifying the maximal number of subjects who are truly frequent ED use patients and (b) minimizing subjects incorrectly flagged as frequent ED use patients, we aimed to balance the cost of incorrectly identifying frequent ED patients by setting the prediction model's sensitivity at $25 \%$. Although the models had PPVs around $60 \%$, a significant proportion of false positive patients actually had more than $8 \mathrm{ED}$ visits in two years. The adjusted PPV for patients having 8 or more visits in the model that predicting frequent ED users as 16 or more visits is $81.9 \%$. To our knowledge, 
this is the first study to employ routine registration data to develop predictive algorithm to predict future frequent ED use. The prediction accuracy strongly suggests that it is feasible to apply routinely collected registration data for future frequent ED utilization prediction.

Limitations of our study include the following: First, we lacked comprehensive population level data for persons who did not use the emergency department. Therefore our analysis is limited to characterizing those individuals who present to emergency departments. Second, we did not include data such as patients' socioeconomic status, since that data is not routinely captured in ED registration data. Third, the applicability of our model to ED registration data from other sites is not assessed, and the predictive performance of the models might be overrated. In the future, we seek to validate this approach against other datasets in a geographically distinct region. Finally, we only evaluated models with $25 \%$ sensitivity as we aimed to balance the cost of ED utilization and the intervention support cost from incorrectly identified frequent ED users.

\section{Conclusions}

We demonstrate a strong association between predictor variables present in routine registration data and frequent ED use. This analysis suggests that it is technically feasible to use routinely collected registration data to identify such use, and the model's observed prediction accuracy may support identifying and intervening to ensure health care resources will be delivered to ensure this group will maximally benefit from intervention. Future work will include validating our algorithm using data sets from other state or organizations within United States.

\section{Competing interests}

The authors have no competing interests to declare.

\section{Authors' contributions}

JW collected the data, conceptualized and designed the study, performed all aspects of data analysis, interpreted the data, wrote and revised the paper critically; HX contributed to study design and data interpretation, reviewed and edited the paper; JF contributed to conception and design, reviewed and edited the paper; SG conceptualized and designed the study, interpreted the data, reviewed and edited the paper critically. All authors read and approved the final manuscript.

\section{Acknowledgements}

We acknowledge James Egg, Joe Kesterson and Jane Wang for their assistance on data extracting and processing.

\section{Funding}

This study is supported in part by the CDC through the Indiana Center of Excellence in Public Health Informatics (1P01HK000077-01) and award (T150C000047-01) from the Office of the National Coordinator for Health Information Technology, Os, Hhs in United States.

\section{Author details}

${ }^{1}$ Regenstrief Institute, Inc., 1101 West 10th Street, Indianapolis, IN 46202, USA. ${ }^{2}$ Department of Biostatistics, Indiana University School of Public Health and School of Medicine, Indianapolis, IN 46202, USA.

Received: 25 July 2014 Accepted: 1 February 2016

Published online: 09 February 2016

\section{References}

1. LaCalle E, Rabin E. Frequent users of emergency departments: the myths, the data, and the policy implications. Ann Emerg Med. 2010;56(1):42-8.

2. Shumway M, Boccellari A, O'Brien K, Okin RL. Cost-effectiveness of clinical case management for ED frequent users: results of a randomized trial. Am J Emerg Med. 2008;26:155-64.

3. Spillane LL, Lumb EW, Cobaugh DJ, Wilcox SR, Clark JS, Schneider SM. Frequent users of the emergency department: can we intervene? Acad Emerg Med. 1997;4:574-80.

4. Lee KH, Davenport L. Can case management interventions reduce the number of emergency department visits by frequent users? Health Care Manag (Frederick). 2006;25:155-9.

5. Kne T, Young R, Spillane L. Frequent ED users patterns of use over time. Am J Emerg Med. 1998;16(7):648-52.

6. Morgan SR, Chang AM, Alqatari M, Pines JM. Non-emergency department interventions to reduce ED utilization: a systematic review. Acad Emerg Med. 2013;20(10):969-85

7. Sun BC, Burstin HR, Brennan TA. Predictors and outcomes of frequent emergency department users. Acad Emerg Med. 2003;10(4):320-8.

8. Zuckerman S, Shen YC. Characteristics of occasional and frequent emergency department users: do insurance coverage and access to care matter? Med Care. 2004;42(2):176-82.

9. Hunt KA, Weber EJ, Showstack JA, Colby DC, Callaham ML. Characteristics of frequent users of emergency departments. Ann Emerg Med. 2006;48(1):1-8.

10. Pines $J M$, Buford $K$. Predictors of frequent emergency department utilization in Southeastern Pennsylvania. J Asthma. 2006;43(3):219-23.

11. Milbrett $P$, Halm M. Characteristics and predictors of frequent utilization of emergency services. J Emerg Nurs. 2009;35(3):191-8.

12. Blank FS, Li H, Henneman PL, Smithline HA, Santoro JS, Provost D, et al. A descriptive study of heavy emergency department users at an academic emergency department reveals heavy ED users have better access to care than average users. J Emerg Nurs. 2005;31 (2):139-44.

13. Locker TE, Baston S, Mason SM, Nicholl J. Defining frequent use of an urban emergency department. Emerg Med J. 2007;24(6):398-401.

14. Doupe MB, Palatnick W, Day S, Chateau D, Soodeen RA, Burchill C, et al. Frequent users of emergency departments: developing standard definitions and defining prominent risk factors. Ann Emerg Med. 2012;60(1):24-32.

15. Fertel BS, Hart KW, Lindsell CJ, Ryan RJ, Lyons MS. Toward understanding the difference between using patients or encounters in the accounting of emergency department utilization. Ann Emerg Med. 2012;60(6):693-8.

16. Roland M, Abel G. Reducing emergency admissions: are we on the right track? BMJ. 2012:8:345-e6017.

17. Finnell JT, Overhage JM, Grannis S. All health care is not local: an evaluation of the distribution of Emergency Department care delivered in Indiana. AMIA Annu Symp Proc. 2011;409:16.

18. Grannis S, Egg J, Ribeka N, RecMatch. Probabilistic Patient Record Matching. 2008,

19. Tsui FC, Espino JU, Dato VM, Gesteland PH, Hutman J, Wagner MM. Technical Description of RODS: A Real-time Public Health Surveillance System. J Am Med Inform Assoc. 2003;10:399-408.

20. Lewis MD, Pavlin JA, Mansfield JL, O'Brien S, Boomsma LG, Elbert Y, et al. Disease Outbreak Detection System Using Syndromic Data in the Greater Washington DC Area. Am J Prev Med. 2002;23(3):180-6.

21. Brillman JC, Burr T, Forslund D, Joyce E, Picard R, Umland E. Modeling emergency department visit patterns for infectious disease complaints: results and application to disease surveillance. BMC Med Inform Decis Mak. 2005;5:4.

22. Moe J, Bailey AL, Oland R, Levesque L, Murray H. Defining, quantifying, and characterizing adult frequent users of a suburban Canadian emergency department. CJEM. 2013;15:1-13.

23. Geurts J, Palatnick W, Strome T, Weldon E. Frequent users of an inner-city emergency department. CJEM. 2012;14(5):306-13. 
24. Fuda KK, Immekus R. Frequent users of Massachusetts emergency departments: a statewide analysis. Ann Emerg Med. 2006;48(1):9-16.

25. Cook LJ, Knight S, Junkins Jr EP, Mann NC, Dean JM, Olson LM. Repeat patients to the emergency department in a statewide database. Acad Emerg Med. 2004;11(3):256-63.

26. South Carolina Public health institute. A Report on Frequent Users of Hospital Emergency Departments in South Carolina. 2011.

27. Kilbreth B, Shaw B, Westcott D, Gray C. Analysis of emergency department use in Maine. January: Muskie School of Public Service; 2010.

28. Bodenmann $\mathrm{P}$, Velonaki VS, Ruggeri $\mathrm{O}$, Hugli $\mathrm{O}$, Burnand $\mathrm{B}$, Wasserfallen $\mathrm{JB}$, et al. Case management for frequent users of the emergency department: study protocol of a randomised controlled trial. BMC Health Serv Res. 2014;14:264

29. Reinius P, Johansson M, Fjellner A, Werr J, Ohlén G, Edgren G. A telephonebased case-management intervention reduces healthcare utilization for frequent emergency department visitors. Eur J Emerg Med. 2013;20(5):327-34.

30. Crane S, Collins L, Hall J, Rochester D, Patch S. Reducing utilization by uninsured frequent users of the emergency department: combining case management and drop-in group medical appointments. J Am Board Fam Med. 2012;25(2):184-91.

31. Hansagi H, Olsson M, Hussain A, Ohlén G. Is information sharing between the emergency department and primary care useful to the care of frequent emergency department users? Eur J Emerg Med. 2008;15(1):34-9.

\section{Submit your next manuscript to BioMed Central} and we will help you at every step:

- We accept pre-submission inquiries

- Our selector tool helps you to find the most relevant journal

- We provide round the clock customer support

- Convenient online submission

- Thorough peer review

- Inclusion in PubMed and all major indexing services

- Maximum visibility for your research

Submit your manuscript at www.biomedcentral.com/submit

) Biomed Central 